\title{
IPS-1 is crucial for DAP3-mediated anoikis induction by caspase-8 activation
}

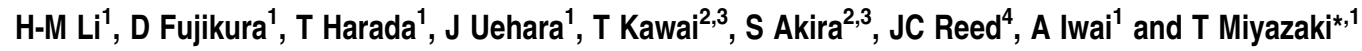

Detachment of adherent epithelial cells from the extracellular matrix induces apoptosis, a process known as anoikis. We have shown that DAP3 is critical for anoikis induction. However, the mechanism for anoikis induction mediated by DAP3 is still unclear. Here, we show that interferon- $\beta$ promoter stimulator 1 (IPS-1) binds DAP3 and induces anoikis by caspase activation. Recently, IPS-1 has been shown to be critical for antiviral immune responses, although there has been no report of its function in apoptosis induction. We show that overexpression of IPS-1 induces apoptosis by activation of caspase-3, -8, and -9. In addition, IPS-1 knockout mouse embryonic fibroblasts were shown to be resistant to anoikis. Interestingly, IPS-1 expression, recruitment of caspase-8 to IPS-1, and caspase-8 activation were induced after cell detachment. Furthermore, DAP3-mediated anoikis induction was inhibited by knockdown of IPS-1 expression. Therefore, we elucidated a novel function of IPS-1 for anoikis induction by caspase-8 activation.

Cell Death and Differentiation (2009) 16, 1615-1621; doi:10.1038/cdd.2009.97; published online 31 July 2009

Apoptosis, the programmed physiological cell death, is critical for modeling tissues and maintaining homeostasis in multicellular organisms. ${ }^{1}$ Two major pathways to induce apoptosis are known as the 'extrinsic' and 'intrinsic' pathways. ${ }^{2,3}$ The extrinsic pathway is triggered by binding of tumor necrosis factor $\alpha(\mathrm{TNF}-\alpha)$ or Fas ligand to cell surface receptors. Binding of these ligands induce recruitment of the adaptor protein, TNF receptor 1-associated death domain protein or Fas-associated death domain (FADD) with procaspases to death receptors, leading to the procaspase activation and apoptosis induction. The intrinsic pathway, also referred to as the mitochondrial apoptosis pathway, responds to intracellular signals induced by DNA damage, cytokine deprivation, or various stimuli. ${ }^{4}$ Activation of this pathway triggers the release into the cytoplasm of proteins that mediate cell death, such as cytochrome $c .{ }^{5}$ Recently, it is shown that multiple pathways activated by intracellular stress signals converge at the mitochondrion to induce loss of outer membrane integrity in a process regulated by $\mathrm{Bcl}-2$ family members. ${ }^{6}$ The extrinsic pathway has also been reported to potentiate the mitochondrial pathway by triggering cytochrome $c$ release through caspase-8-cleaved Bid protein, a pro-apoptotic member of the Bcl-2 family that translocates to the mitochondria. ${ }^{7,8}$ Recently, DAP3 is shown to be essential for mitochondrial homeostasis and contributing to the extrinsic pathway for apoptosis induction. ${ }^{9}$

Adhesion to an appropriate extracellular matrix is essential for the survival of many normal cells, and loss of adhesion induces programmed cell death, a phenomenon known as anoikis. Anoikis was first observed in endothelial and epithelial cells, in which loss of matrix attachment, even in the presence of serum, induces cell death. Expression of certain oncogenes can provide protection from anoikis, ${ }^{10-13}$ leading to metastasis induction. We have shown that deathassociated protein 3 (DAP3) is critical for anoikis induction through interaction with FADD, correlating with caspase-8 activation. ${ }^{14}$ Downregulation of DAP3 expression inhibited anoikis and overexpression of DAP3 augmented cell death through caspase activation after cell detachment. Furthermore, association of DAP3 with FADD and caspase-8 activation were induced by cell detachment. Interferon- $\beta$ promoter stimulator 1 (IPS-1), also known as mitochondrial antiviral signaling protein, virus-induced signaling adaptor, and caspase recruitment domain (CARD) adaptor inducing IFN- $\beta$ (Cardif), was recently identified as an adaptor linking RIG-I and Mda5 to the downstream signaling molecules. ${ }^{15-19}$ IPS-1 contains the CARD-like domain that is responsible for the interaction with RIG-I or Mda5. In addition, IPS-1 contains a transmembrane region that targets this protein to the mitochondrial outer membrane. ${ }^{19}$ The mitochondrial localization of IPS-1 is essential for triggering downstream signals, including FADD and caspase-8. ${ }^{19,20}$ However, the functional role of IPS-1 in apoptosis or anoikis induction is not reported.

Here, we report that IPS-1 is associated with DAP3 and that IPS-1 expression is induced after cell detachment.

\footnotetext{
${ }^{1}$ Department of Bioresources, Hokkaido University Research Center for Zoonosis Control, Sapporo, Hokkaido, Japan; ${ }^{2}$ Laboratory of Host Defense, WPI Immunology Frontier Research Center, Osaka University, 3-1 Yamada-oka, Suita, Osaka, Japan; ${ }^{3}$ Department of Host Defense, Research Institute for Microbial Diseases, Osaka University, 3-1 Yamada-oka, Suita, Osaka, Japan and ${ }^{4}$ Burnham Institute for Medical Research, 10901 N. Torrey Pines Rd, La Jolla, CA, USA

${ }^{*}$ Corresponding author: T Miyazaki, Department of Bioresources, Hokkaido University Research Center for Zoonosis Control, Kita-20, Nishi-10, Kita-ku, Sapporo, Hokkaido 001-0020, Japan. Tel: + 8111706 7314; Fax: +81 11706 7314; E-mail: miyazaki@czc.hokudai.ac.jp

Keywords: IPS-1; DAP3; anoikis; casapse-8; ECM

Abbreviations: ECM, extracellular matrix; DAP3, death-associated protein 3; IPS-1, interferon- $\beta$ promoter stimulator 1 ; MEF, mouse embryonic fibroblast; TNF- $\alpha$, tumor necrosis factor $\alpha$; TRADD, TNF receptor 1-associated death domain protein; FADD, FAS-associated death domain; TRAIL, TNF-related apoptosis inducing ligand. Received 05.12.08; revised 18.6.09; accepted 22.6.09; Edited by HU Simon; published online 31.7.09
} 
Overexpression of IPS-1 induced apoptosis and the cleavage of caspase-3, -8, and -9. In addition, IPS-1 knockout (KO) mouse embryonic fibroblasts (MEFs) were resistant to anoikis and activation of caspase-3, -8 , and -9 after cell detachment was reduced in these cells. Conversely, apoptosis induction by death receptor stimulation was not inhibited in IPS-1 KO MEFs. Furthermore, caspase-8 was recruited to IPS-1 and activated after cell detachment. Therefore, we have elucidated a novel function of IPS-1 to activate caspase-8 for anoikis induction.

\section{Results}

IPS-1 is associated with DAP3 and induces caspasedependent apoptosis. To analyze the downstream signal of DAP3, we screened its binding proteins and identified IPS-1 to bind it (Figure 1a; Supplementary Figure 1). Expression of IPS-1 by transfection of its expression vector induced morphological change in the cell shape, detachment, and cell death in 293T cells (Figure 1b). In addition, DNA fragmentation (Figure 1c) and caspase-3, -8 , or -9 activation (Figure 1d) were detected by the expression of IPS-1. Next, we clarified the critical region of IPS-1 to induce apoptosis using expression vector plasmids for deletion mutants of IPS-1, such as IPS-1N (the N-terminal CARD), IPS-1C (the C-terminal non-CARD region), and IPS-1 $\Delta$ Mito (deletion of mitochondrial membrane region) (Figure 1e; Supplementary Figure 2). Expression of IPS-1C but not IPS-1N and IPS-1 $1 \Delta$ Mito induced apoptosis, indicating that the mitochondrial membrane region of IPS-1 is critical for apoptosis induction (Figure 1e).

IPS-1 KO MEF cells are resistant to anoikis by reduced activation of caspase-3, -8 , and -9 after cell detachment. We investigated whether IPS-1 is involved in apoptosis signals mediated by death receptors. After IPS-1 $\mathrm{KO}^{21}$ (Figure 2a) and WT (wild type) MEFs were stimulated with TNF- $\alpha$, TRAIL, or anti-Fas Ab in combination with cycloheximide, cells were analyzed for apoptosis induction. No significant difference for caspase activation or apoptosis induction was observed by the stimulation of these death receptors between IPS-1 KO and WT MEFs (Supplementary Figure 3).

Next, we investigated whether IPS-1 is involved in anoikis induction. At $24 \mathrm{~h}$ after IPS-1 KO or WT MEF cells were detached and cultured in suspension, caspase-3/7 activity in these cells was analyzed. Activity of caspase- $3 / 7$ induced by cell detachment in IPS-1 KO MEFs was lower than that in WT MEFs (Figure 2b). Furthermore, anoikis induction was inhibited in IPS-1 KO MEFs in comparison with WT MEFs at $24 \mathrm{~h}$ after cell detachment analyzed by DNA fragmentation ELISA assay (Figure 2c). In addition, we examined caspase-8 activity in IPS-1 KO and WT MEFs before and after cell detachment. It was shown that caspase-8 activity was lower in IPS-1 KO MEFs than in WT MEFs after cell detachment (Figure 2d). In the same experiment, caspase-9 activity was also lower in IPS-1 KO MEFs than in WT MEFs (Supplementary Figure 4).
DAP3-mediated anoikis induction was inhibited by knockdown of IPS-1 expression. We examined the functional relationship of IPS-1 with DAP3 for anoikis induction. Knockdown of IPS-1 expression by siRNA transfection inhibited anoikis induction (Figure 3a; Supplementary Figure $5 \mathrm{a}$ and b). DAP3-mediated anoikis induction was suppressed by knockdown of IPS-1 expression in 293T cells (Figure 3b; Supplementary Figure 5c). Conversely, we examined whether IPS-1-mediated anoikis induction is inhibited by the knockdown of DAP3 expression $^{9}$ for anoikis induction. It was shown that IPS-1induced apoptosis was not suppressed by the knockdown of DAP3 expression (Supplementary Figure 6). In addition, mutant of DAP3, DAP3(T237E) could be associated with IPS-1 (Supplementary Figure 7).

IPS-1 interacts with caspase- 8 and induces its activation after cell detachment. We examined whether caspase-8 interacts with IPS-1 using 293T cells. We have shown that caspase-8 associated with IPS-1 and the mitochondrial membrane region of IPS-1 was necessary for its association (Figure 4a). Furthermore, we performed immunoprecipitation experiments for the analysis of the interaction of IPS-1 with FADD and caspase-8.

Both FADD and caspase-8 associated with IPS-1, and casapse-8 strongly associated with IPS-1 as compared with FADD (Figure 4b).

IPS-1 is upregulated after cell detachment and induces anoikis. To determine whether expression of IPS-1 is regulated during anoikis induction, we analyzed the expression of IPS-1 in MCF-10A cells, in which apoptosis was induced at both 48 and $72 \mathrm{~h}$ after cell detachment. IPS-1 expression was induced and reached to maximal level at $48 \mathrm{~h}$ after detachment (Figure 5a).

We next examined whether caspase- 8 is recruited to IPS-1 in the mitochondria after cell detachment. We found that caspase-8 was co-immunoprecipitated with IPS-1 in a mitochondrial fraction after cell detachment (Figure $5 b$ ).

\section{Discussion}

Recent studies suggest that DAP3, a pro-apoptotic protein, is required for mitochondrial homeostasis in vivo and contributes to the extrinsic pathway for apoptosis. ${ }^{9}$ It has been shown that DAP3 is important for apoptosis induction. ${ }^{14,22,23}$ However, the mechanism for DAP3-mediated apoptosis induction is still unknown. Therefore, we screened its binding proteins and identified IPS-1 as a DAP3-binding protein (Figure 1a; Supplementary Figure 1). As shown in Figure 1b, expression of IPS-1 induced cell morphological change, detachment, and cell death. As DNA fragmentation (Figure 1c) and caspase-3, -8 , or -9 activation (Figure 1d) were detected, it is suggested that IPS-1 expression induces apoptosis by caspase activation. Next, to identify the critical region of IPS-1 for apoptosis induction, we examined the function of some IPS-1 mutants. As expression of IPS-1C but not IPS-1N and IPS-1 $1 \Delta$ Mito induced apoptosis, it is suggested that the mitochondrial membrane region of IPS-1 is critical for apoptosis induction (Figure 1e; Supplementary Figure 2). 


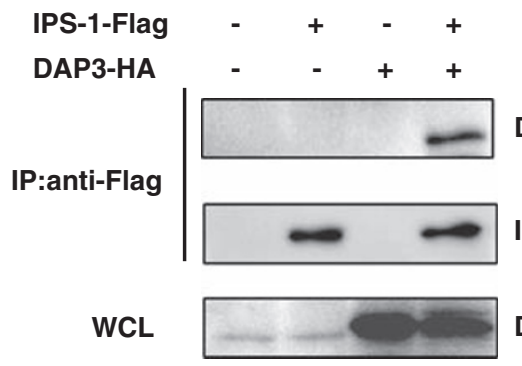

DAP3

IPS-1

DAP3
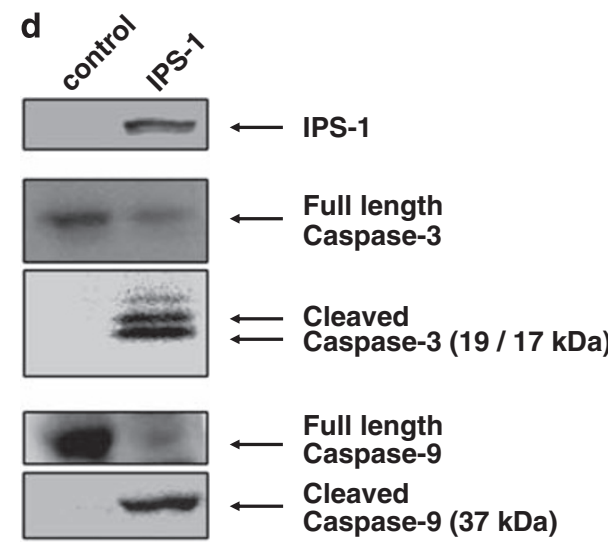

Caspase-9 (37 kDa)
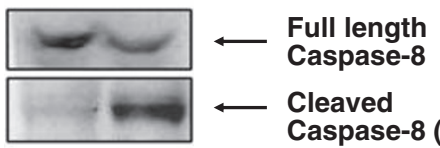

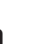

b

control
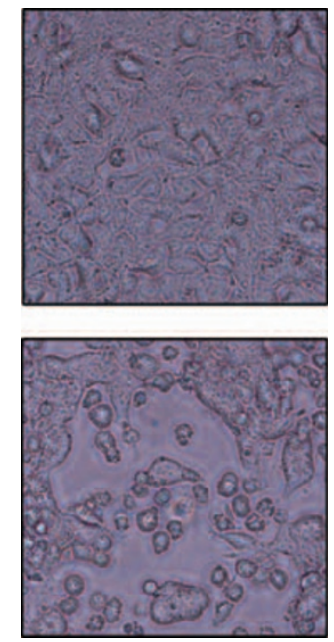
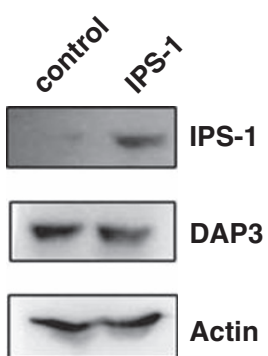

C

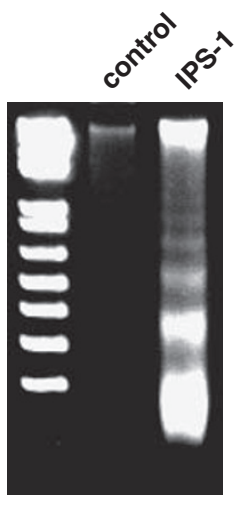

e

\begin{tabular}{|c|c|}
\hline IPS-1 FL & \begin{tabular}{|l|l|} 
CARD & Pro \\
\end{tabular} \\
\hline IPS-1 N & CARD \\
\hline IPS-1 C & \begin{tabular}{|l|} 
Pro \\
\end{tabular} \\
\hline IPS-1 $1 \Delta$ Mito & \begin{tabular}{|l|l|} 
CARD & Pro \\
\end{tabular} \\
\hline
\end{tabular}

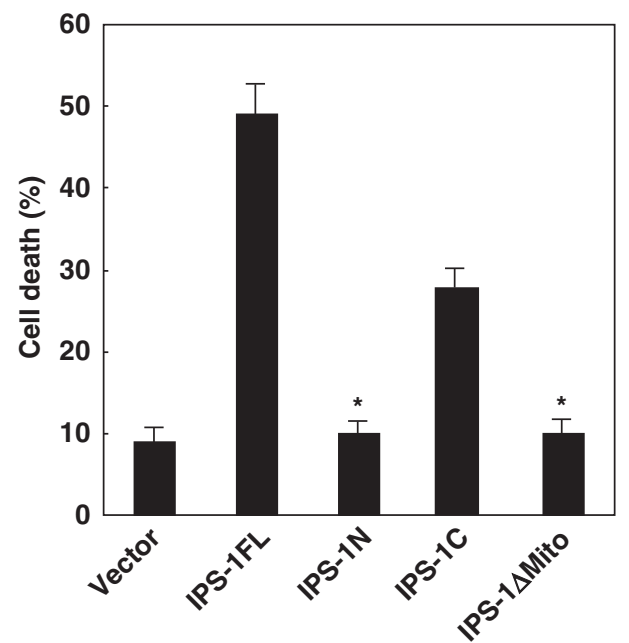

Figure 1 IPS-1 associates with DAP3 and expression of IPS-1 induces apoptosis. (a) IPS-1 is associated with DAP3. Expression vector plasmids for IPS-1 and DAP3 were transfected into HEK 293T cells. The cell lysates were immunoprecipitated with anti-Flag antibody, analyzed by SDS-PAGE and immunoblotted with anti-Flag (for the detection of IPS-1) or anti-HA (for the detection of DAP3) antibody. Whole cell lysates (WCL) were analyzed by SDS-PAGE and immunoblotted with anti-HA antibody (for the detection of DAP3). (b) Expression of IPS-1 induces cell detachment and cell death. HEK 293T cells were transfected with the expression vector for control vector or IPS-1 expression vector and were photographed at $48 \mathrm{~h}$ after the transfection under a microscope (left). Whole cell lysates were analyzed by SDS-PAGE and immunoblotted with anti-IPS-1, anti-DAP3, or anti- $\beta$-actin antibody (right). (c) Expression of IPS-1 induces DNA fragmentation. The cells were transfected with control vector or IPS-1 expression vector and were collected at $48 \mathrm{~h}$ after the transfection. Extracted genomic DNA from these cells was analyzed by agarose gel electrophoresis. A typical DNA ladder was detected in the cells transfected with only IPS-1 expression vector. (d) Expression of IPS-1 induces the cleavage of caspase-3, -9 , and -8 . HEK 293T cells were transfected with the expression vector for IPS-1 or control vector and were collected at $48 \mathrm{~h}$ after the transfection. The cell lysates were analyzed by western blot analysis using anti-Flag (for the detection of IPS-1), anti-cleaved, or full length of caspase-3, caspase-9, and caspase-8 or anti- $\beta$-actin antibody as a control. (e) Mitochondrial membrane region of IPS-1 is critical for the induction of cell death. Expression vectors for IPS-1FL (IPS-1 full length), IPS-1N (the N-terminal CARD alone), IPS-1C (the C-terminal non-CARD region), or IPS-1 $\triangle$ Mito (lacking mitochondrial membrane region) were transfected into HEK 293T cells. The percentage of dead cells was assayed by trypan blue staining at $48 \mathrm{~h}$ after the transfection. Data represent the means \pm S.D. for the data of four independent experiments. Asterisks indicate the statistical significance, as the $P$-value is lower than 0.05 compared with the data for the cells transfected with the expression vector of IPS-1FL 


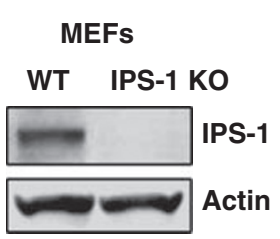

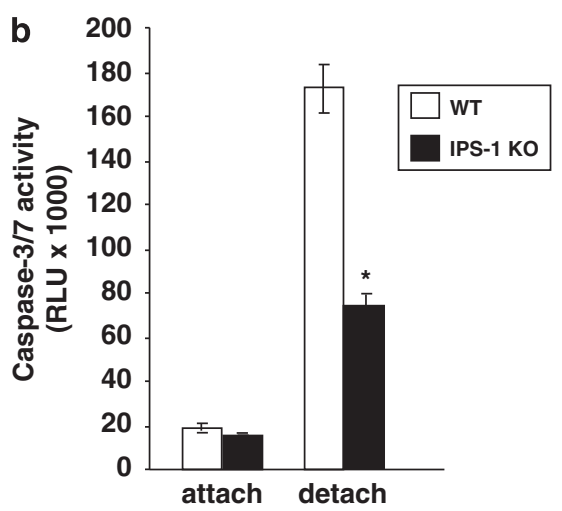

C

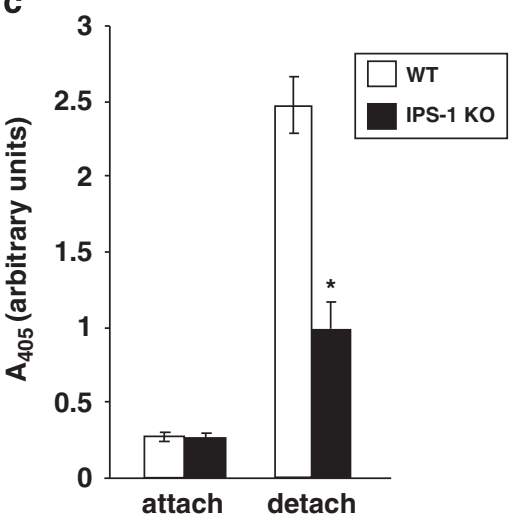

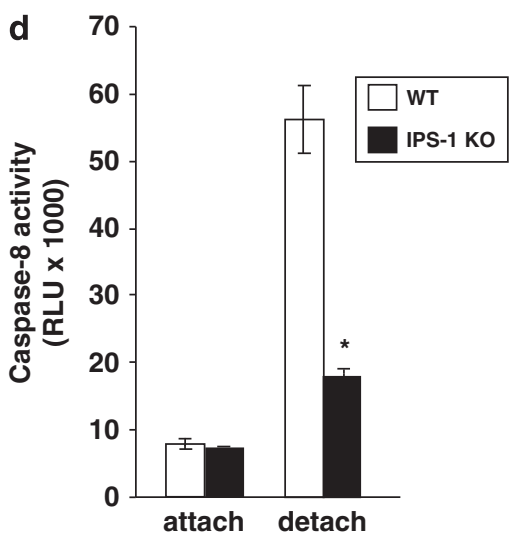

Figure 2 IPS-1 knockout (KO) cells are resistant to anoikis induction. (a) Expression of IPS-1 protein in WT or IPS-1 KO mouse embryonic fibroblasts (MEFs). WT and IPS-1 KO MEFs were lysed, analyzed by SDS-PAGE, and immunoblotted with anti-IPS- 1 or anti- $\beta$-actin antibody. (b) Caspase-3/7 activity is lower in IPS-1 KO cells than in WT cells after cell detachment. WT or IPS-1 KO MEFs were cultured in the attached or detached (in suspension culture) condition. After $24 \mathrm{~h}$, these cells were collected and analyzed for caspase- $3 / 7$ activity. Data represent the means \pm S.D. for the data of four independent experiments. Asterisks indicate the statistical significance, as the $P$-value is lower than 0.05 compared with the data for detached WT cells. (c) DNA fragmentation is inhibited in IPS-1 KO cells as compared with WT cells after cell detachment. WT or IPS-1 KO MEFs were cultured in the attached or detached (in suspension culture) condition. After $24 \mathrm{~h}$, these cells were collected and analyzed for DNA fragmentation ELISA assay. Data represent the means \pm S.D. for the data of four independent experiments. Asterisks indicate the statistical significance, as the $P$-value is lower than 0.05 compared with the data for detached WT cells. (d) Caspase-8 activity is lower in IPS-1 KO cells than in WT cells after cell detachment. WT and IPS-1 KO MEFs were cultured in the attached or detached (in suspension culture) condition. At $8 \mathrm{~h}$ after the detachment, caspase-8 activity was assessed using caspase-8 Glo assay reagents. Data represent the means \pm S.D. for the data of four independent experiments. Asterisks indicate the statistical significance, as the $P$-value is lower than 0.02 compared with the data for detached WT cells

IPS-1 interacts with FADD, caspase-8, and $-10 .{ }^{20}$ As shown in Figure 1d, we found that overexpression of IPS-1 cleaves caspase- $3,-8$, and -9 . Therefore, we examined whether IPS-1 is critical for apoptosis signals mediated by death receptors. IPS-1 $\mathrm{KO}^{21}$ (Figure 2a) and WT MEFs were stimulated with TNF- $\alpha$, TRAIL, or anti-Fas Ab in combination with cycloheximide but there was no significant difference for caspase activation or apoptosis induction (Supplementary Figure 3). These results show that IPS-1 is not critical for apoptosis induction mediated by death receptors.

We have shown earlier that DAP3 is critical for anoikis induction. ${ }^{14}$ Therefore, we next examined whether IPS-1 is critical for anoikis induction. After IPS-1 KO or WT MEF cells were detached and cultured in suspension, caspase-3/7 activity of IPS-1 KO MEFs was lower than that of WT MEFs (Figure 2b). It is shown that IPS-1 is involved in caspase-3/7 activation after cell detachment. Furthermore, anoikis induction was inhibited in IPS-1 KO MEFs in comparison with WT MEFs after cell detachment (Figure 2c). These results indicate that IPS-1 is critical for anoikis induction.
Casapase-8 activation is an initiating event in anoikis induction. ${ }^{24,25}$ Therefore, we examined caspase-8 activity and it was shown to be lower in IPS-1 KO MEFs than in WT MEFs after cell detachment (Figure 2d). As shown in Supplementary Figure 4, caspase-9 activity was also lower in IPS-1 KO MEFs than in WT MEFs. These results show that IPS-1 is critical for caspase-3, -8 , and -9 activation after cell detachment.

Knockdown of IPS-1 expression by siRNA inhibited anoikis induction in 293T cells (Figure 3a; Supplementary Figure 5a and $b$ ). We have shown earlier that DAP3 was critical for anoikis induction, ${ }^{14}$ and we show here that DAP3 associates with IPS-1 (Figure 1a; Supplementary Figure 1). Therefore, we evaluated the functional relationship of IPS-1 with DAP3 for anoikis induction. DAP3-mediated anoikis induction was suppressed by knockdown of IPS-1 expression (Figure 3b; Supplementary Figure 5c). Conversely, we examined whether IPS-1-mediated anoikis induction is inhibited by the knockdown of DAP3 expression. IPS-1-induced apoptosis was shown not to be suppressed by the knockdown of DAP3 expression (Supplementary Figure 6). These results indicated 
a

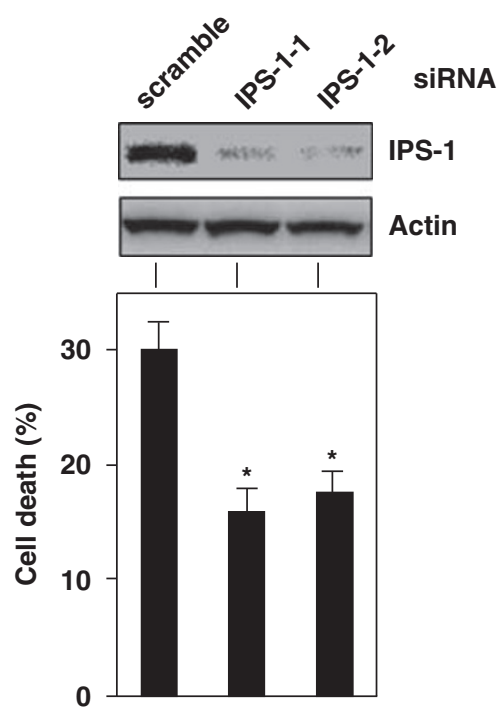

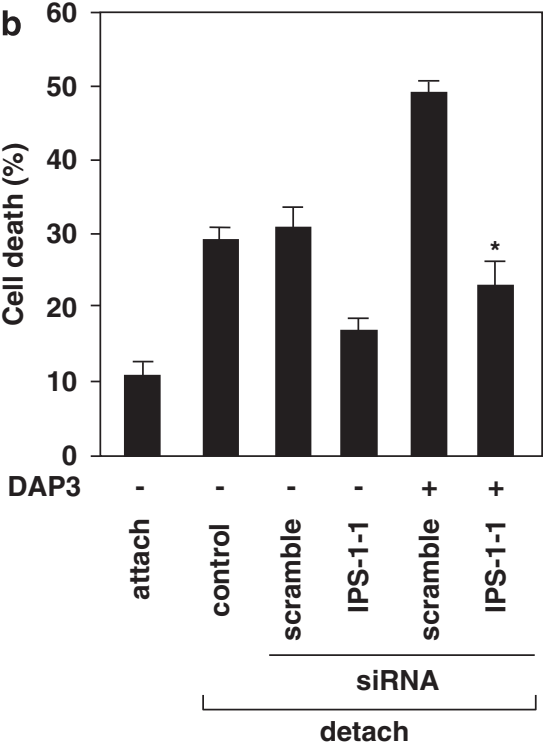

Figure 3 Knockdown of IPS-1 expression inhibits DAP3-mediated anoikis induction. (a) IPS-1 expression is inhibited by siRNA transfection (upper part). HEK 293 cells were transfected with two different siRNAs, IPS-1-1 and IPS-1-2 for IPS-1 knockdown or scramble siRNA (negative control). These cell lysates were analyzed by SDS-PAGE and immunoblotted with anti-IPS-1 or anti- $\beta$-actin antibody. Knockdown of endogenous IPS-1 expression inhibits cell death after the detachment (lower part). HEK 293 cells transfected with siRNA were cultured in poly-HEMA-coated plates for $24 \mathrm{~h}$, and the percentage of dead cells was assayed by trypan blue staining. Data represent the means \pm S.D. for the data of four independent experiments. Asterisks indicate the statistical significance, as the $P$-value is lower than 0.05 compared with the data for cells transfected with scramble siRNA. (b) DAP3-mediated anoikis induction is inhibited by knockdown of IPS-1 expression. HEK 293 cells were transfected with scramble or IPS-1-1 siRNA together with expression vector plasmid encoding DAP3 or control vector. After $24 \mathrm{~h}$, the transfected cells were detached and cultured in HEMA-coated dishes for $24 \mathrm{~h}$ (detach) or in attached condition (attach). The percentage of dead cells was assayed by trypan blue staining. Data represent the means \pm S.D. for the data of three independent experiments. Asterisks indicate the statistical significance, as the $P$-value is lower than 0.05 compared with the data for cells transfected with DAP3 and scramble siRNA

that IPS-1 is located at the downstream of DAP3 in anoikis signal. DAP3(T237E), which is the phospho-mimic mutant, ${ }^{14}$ could be associated with IPS-1 (Supplementary Figure 7), the phosphorylation of DAP3 by Akt is not involved in the interaction of DAP3 with IPS-1.

It was shown that IPS-1 interacts with FADD, ${ }^{16}$ and that overexpression of IPS-1 induced apoptosis (Figure 1b and c) and cleavage of caspase-8 (Figure 1d). Therefore, we examined the interaction of caspase- 8 with IPS- 1 and caspase-8 was shown to be associated with IPS-1 (Figure 4a). In addition, the mitochondrial membrane region of IPS-1 was shown to be essential for its interaction. Furthermore, we examined the interaction of IPS-1 with FADD and caspase-8. As shown in Figure 4b, both FADD and caspase-8 were associated with IPS-1. It is also shown that casapse-8 was strongly associated with IPS-1 than FADD. These results suggest that IPS-1 induces anoikis by the interaction with caspase-8 and FADD to activate caspase-8.

To evaluate whether IPS-1 expression is regulated during anoikis induction, we analyzed its expression before and after cell detachment. As shown in Figure 5a, IPS-1 expression was induced after detachment and Bim expression was also induced as shown earlier. ${ }^{26}$ As IPS-1 KO MEF cells are resistant to anoikis, it is suggested that upregulation of IPS-1 expression during detachment contributes to anoikis induction.

Detachment of the MDCK and MCF-10A cells led to the activation of caspase-8, which triggered mitochondrial dysfunction and activation of the effector caspase- 3 and $-7.24,25$ We found that caspase-8 was associated with IPS-1 and
IPS-1 expression induced the cleavage of caspase-8 (Figures $1 \mathrm{~d}, 4 \mathrm{a}$ and $\mathrm{b}$ ). Therefore, we next examined whether the interaction of caspase-8 with IPS-1 is induced in the mitochondria after cell detachment. As shown in Figure 5b, procaspase-8 was associated with IPS-1 in a mitochondrial fraction after cell detachment.

In conclusion, IPS-1 was shown to be crucial for anoikis induction at the downstream of DAP3 by caspase- 8 activation after cell detachment.

\section{Materials and Methods}

Cell culture and materials. MCF-10A cells were cultured as described earlier. ${ }^{26}$ HEK293, 293T cells, or MEFs from IPS-1-deficient mice were maintained in DMEM supplemented with $10 \% \mathrm{FCS}$, penicillin $(50 \mathrm{U} / \mathrm{ml})$, and streptomycin $(50 \mu \mathrm{g} / \mathrm{ml})$. IPS-1-deficient mice were generated by the standard gene targeting. ${ }^{21}$ The targeting vector was designed to disrupt two exons harboring the CARD-like domain of IPS-1, which is required for its function. Immunoprecipitation and immunoblotting were performed using anti-HA (Roche, Basel, Switzerland), anti-flag (Sigma, St Louis, MO, USA) antibody. Anti-Bim, anti-cleaved caspase-3, anticleaved caspase-8, anti-cleaved caspase-9 antibodies were purchased from Cell Signaling Technology, Danvers, MA, USA. Anti-FADD antibody (BD Transduction Laboratories, Franklin Lakes, NJ, USA), anti-IPS-1 (Cardif) antibody (Alexis Biochemicals, Rosen, Switzerland), anti- $\beta$-actin antibody (Chemicon, Temecula, CA, USA), and anti-mouse IPS-1 antibody (Kumar et al., 2006) were used for the western blotting analysis.

Transfections, immunoprecipitation, and immunoblotting. HEK293 or 293T cells $\left(1 \times 10^{6}\right)$ in $10 \mathrm{~cm}$ culture dishes were transiently transfected with $10 \mu \mathrm{g}$ (total) of plasmid DNA with lipofectamine 2000 . After $48 \mathrm{~h}$, the cells were collected and suspended in lysis buffer containing $0.5 \%$ Nonidet P-40, $20 \mathrm{mM}$ Tris$\mathrm{HCl}$ buffer (pH 7.5), $1 \mathrm{mM}$ EDTA, $150 \mathrm{mM} \mathrm{NaCl}$, and protease inhibitor (Complete, Mini). Immunoprecipitations were performed using $10 \mu \mathrm{l}$ of anti-FLAG antibody M2- 


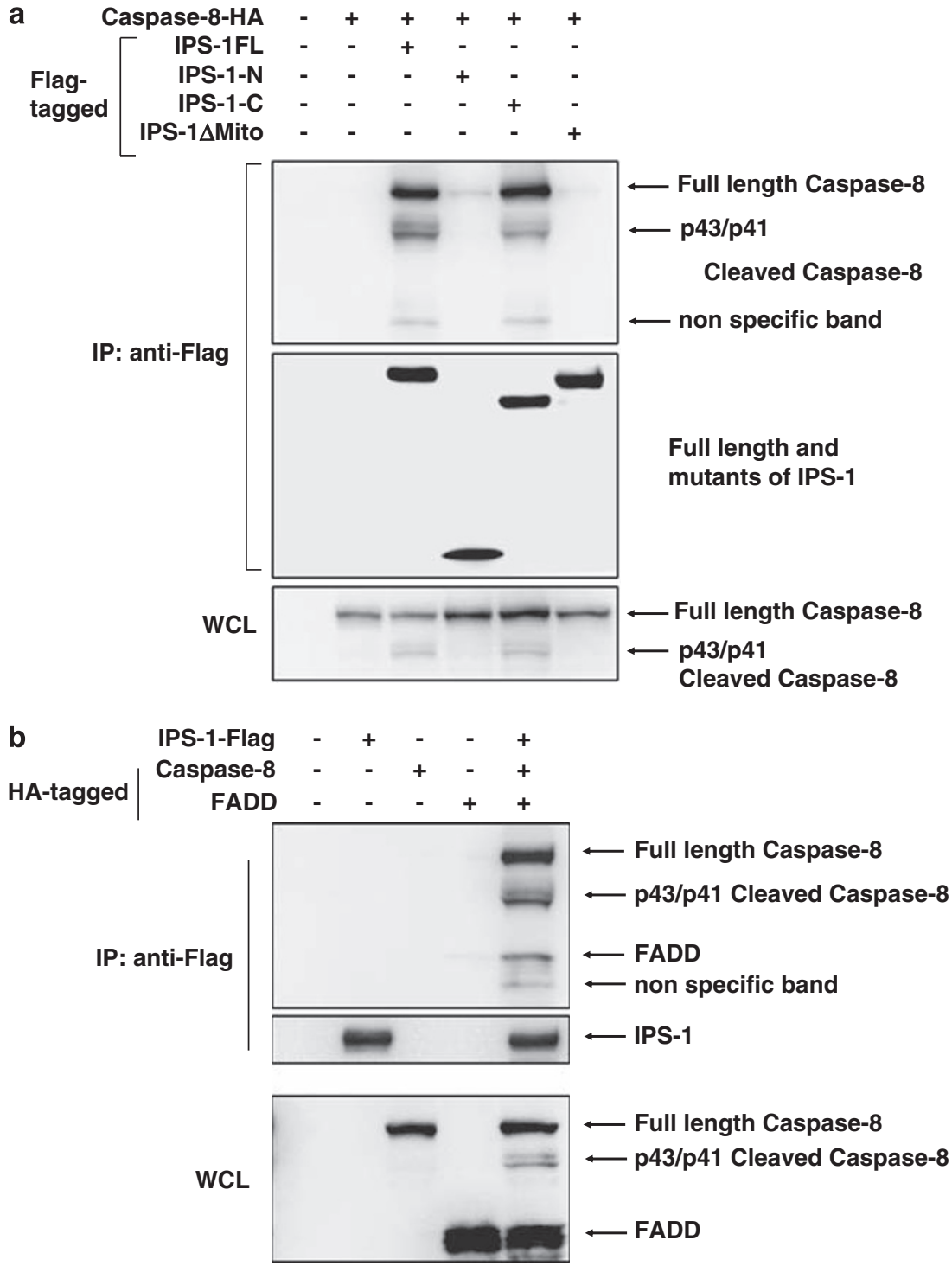

Figure 4 IPS-1 binds to caspase-8 and induces its activation. (a) Mitochondrial membrane region of IPS- 1 is critical for the association with caspase-8 and its activation. Expression vector plasmids for Flag-tagged IPS-1FL, IPS-1N, IPS-1C, IPS-1 $\Delta$ Mito, and HA-caspase-8 were transfected into HEK 293T cells with the indicated combination, their cell lysates were immunoprecipitated with anti-Flag antibody and immunoblotted with anti-HA or anti-Flag antibody. Whole cell lysates (WCL) were analyzed by SDSPAGE and immunoblotted with anti-HA antibody. (b) IPS-1 strongly binds to caspase-8 as compared with FADD. After the expression vector plasmids for Flag-IPS-1, HA-caspase-8, and HA-FADD were transfected into HEK 293T cells, their cell lysates were immunoprecipitated with anti-Flag antibody and immunoblotted with anti-HA or anti-Flag antibody. Whole cell lysates (WCL) were analyzed by SDS-PAGE and immunoblotted with anti-HA antibody

conjugated-agarose (Sigma) or the other antibodies at $4{ }^{\circ} \mathrm{C}$ for $2 \mathrm{~h}$ after the cell lysates were precleared with $10 \mu \mathrm{l}$ of protein A-agarose or protein G-agarose. The immune complexes were analyzed by SDS-PAGE and immunoblotted using the indicated antibodies after extensive washing by lysis buffer. MCF-10A cells were transfected with siRNA (20 nM) using oligofectamine $(16 \mu \mathrm{l})$. At first, OPTI-MEM $(500 \mu \mathrm{l})$ and oligofectamine were mixed and incubated at room temperature for $5 \mathrm{~min}$. This solution was added to the solution including OPTI-MEM $(500 \mu l)$ and each siRNA. Finally, this mixed solution was incubated at room temperature for $20 \mathrm{~min}$ and added to each well in which MCF-10A cells were cultured. These cells were cultured with growth medium containing $0.5 \%$ methylcellulose (Sigma) in poly-HEMA-coated culture plates for the indicated times. Cell lysates were analyzed by immunoblotting with antibodies for IPS-1, Bim, or $\beta$-actin. For the immunoprecipitation, cytosolic and mitochondrial fractions were prepared from MCF-10A cells $\left(5 \times 10^{7}\right)$ in the attached condition or in suspension culture for $6 \mathrm{~h}$, using the mitochondrial/cytosol fractionation kit (Alexis Biochemicals). Mitochondrial lysates were immunoprecipitated with rabbit anti-human IPS-1 antibody $(2 \mu \mathrm{g})$ and protein A/G-Sepharose beads overnight at $4^{\circ} \mathrm{C}$.
Immunoprecipitated proteins were analyzed by western blotting using antibodies for caspase-8 or IPS-1.

DNA fragmentation assay. After cells $\left(5 \times 10^{5}\right)$ were suspended in $100 \mu \mathrm{l}$ of $10 \mathrm{mM}$ Tris- $\mathrm{HCl}(\mathrm{pH} 7.5)$ buffer containing $10 \mathrm{mM}$ EDTA, $0.5 \%$ Triton $\mathrm{X}-100$, and RNase A $(200 \mu \mathrm{g} / \mathrm{ml})$, cell lysates were incubated for $10 \mathrm{~min}$ at the room temperature. These cell lysates were centrifuged at $1.5 \times 10^{4}$ r.p.m. for $20 \mathrm{~min}$ at $4{ }^{\circ} \mathrm{C}$, the supernatant was incubated for $1 \mathrm{~h}$ at $37^{\circ} \mathrm{C}$. Then, $1 \mu$ l of proteinase $\mathrm{K}$ $(20 \mathrm{mg} / \mathrm{ml})$ was added, and the solution was incubated for additional $1 \mathrm{~h}$. DNA was precipitated with $50 \%$ isopropanol containing $420 \mathrm{mM} \mathrm{NaCl}$, dissolved in TE solution, separated in $2 \%$ agarose gels, and stained with ethidium bromide. The stained gels were photographed on a UV transilluminator.

Assay for the activity of caspase-3/7, caspase-8, or -9. Activity of caspase-3/7, caspase-8, or -9 was measured using caspase-Glo $3 / 7$, caspase-Glo 8 , or caspase-Glo 9 detection assay kit (Promega, Madison, WI, USA). These 


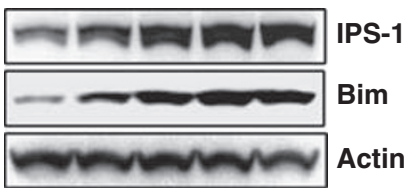

b

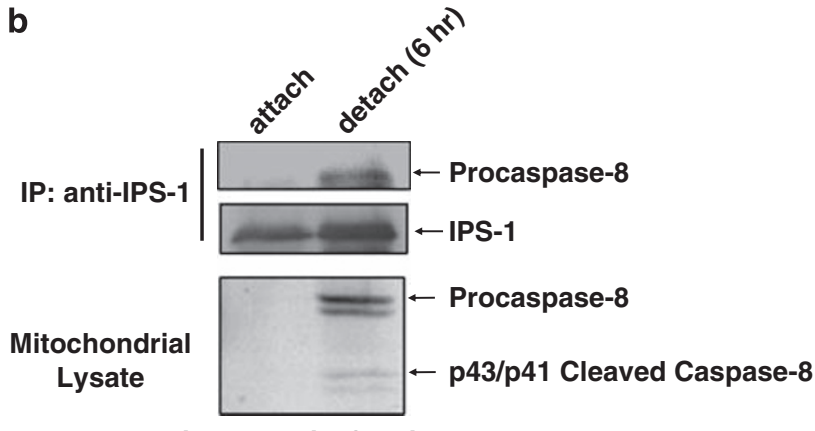

Mitochondrial fraction

Figure 5 IPS-1 expression is upregulated after cell detachment and contributes to anoikis induction. (a) Lack of attachment induces the upregulation of IPS-1 expression. MCF-10A cells were detached and cultured in poly-HEMA-coated plates with growth medium for the indicated period of time. The cell lysates were analyzed by SDS-PAGE and immunoblotted with antibodies for IPS-1, Bim- $E_{L}$, or $\beta$-actin. (b) Caspase-8 is recruited to IPS-1 after cell detachment. Mitochondrial fractions were prepared from MCF-10A cells $\left(2 \times 10^{7}\right)$ in attached or detached (cultured in suspension for $6 \mathrm{~h}$ ) condition. Mitochondrial fraction lysates were immunoprecipitated with an anti-IPS-1 antibody, analyzed by SDS-PAGE and immunoblotted with anticaspase-8 or anti-IPS-1 antibody. Whole mitochondrial fraction was analyzed by SDSPAGE and immunoblotted with anti-caspase-8 antibody

reagents (Promega) were equilibrated to the room temperature and mixed well. Each reagent $(100 \mu \mathrm{l})$ was added to each well of 96-well plates, in which attached cells or detached cells were cultured. Each plate was gently mixed and incubated at room temperature for $30 \mathrm{~min}$ to $1 \mathrm{~h}$. The luminescence of each sample was measured by a plate-reading luminometer. Wt and IPS-1 KO MEFs were seeded into 96 -well plates at a density of $5 \times 10^{3}$ cells/well in triplicate wells. After $24 \mathrm{~h}$, cells were treated with $10 \mu \mathrm{g} / \mathrm{ml}$ cycloheximide (CHX) alone or in combination with TNF- $\alpha$ $(0.05 \mu \mathrm{g} / \mathrm{ml})$, anti-Fas Antibody $(\mathrm{Jo2}, 200 \mathrm{ng} / \mathrm{ml})$, or TRAIL $(0.2 \mu \mathrm{g} / \mathrm{ml})$. After these stimulations, caspase- $3 / 7$ activity was assessed by caspase- $3 / 7$ Glo reagents.

Anoikis assays. Anoikis assays were performed using established procedures. ${ }^{11}$ WT and IPS-1 KO MEFs were seeded into the wells of 96-well plates with or without poly-HEMA-coating at a density of $5 \times 10^{3}$ cells/well in triplicate wells. Cells after detachment were cultured in $1 \%$ FBS medium with methylcellulose $(0.5 \%)$. Cells $\left(2.5 \times 10^{4}\right)$ were analyzed for apoptosis induction using the cell death detection ELISA kit (Roche). The analysis at each time point was performed in duplicate and each experiment was carried out at least three times. For the MTT assay, cells were cultured in 96 well-plates for $24 \mathrm{~h}$. Then, $10 \mu \mathrm{l}$ of MTT assay solution of Cell Counting Kit-8 (Wako Chem. Osaka, Osaka, Japan) was added to each well and after $3 \mathrm{~h}$ the absorbance of the solution was measured by spectrophotometer.

Plasmids and RNAi. Flag-tagged and myc-tagged expression vectors for human IPS-1 and mutants (IPS-1N, IPS-1C, and IPS-1 $\Delta$ Mito) were used as described earlier. ${ }^{16}$ Flag-tagged and HA-tagged expression vectors for human DAP3, FADD, and caspase-8 were used as described earlier. ${ }^{23}$ For siRNA experiments, double-stranded RNA duplexes composed of 21-nucleotide sense and antisense oligonucleotides were synthesized by iGENE and used as described earlier. ${ }^{16}$ Knockdown of IPS-1 expression was verified by western blotting analysis using anti-IPS-1 antibody.
Acknowledgements. This work was supported by Grants-in-Aid for Scientific Research from Ministry of Education, Culture, Sports, Science \& Technology, Grants from Japan Health Sciences Foundation, and Grant of the Program of Founding Research Centers for Emerging and Reemerging Infectious Diseases, MEXT Japan and Grants by the National Institutes of Health (GM61694 and Al5624).

1. Jacobson MD, Weil M, Raff MC. Programmed cell death in animal development. Cell 1997; 88: $347-354$.

2. Wajant H. The Fas signaling pathway: more than a paradigm. Science 2002; 296 : 1635-1636

3. Opferman JT, Korsmeyer SJ. Apoptosis in the development and maintenance of the immune system. Nat Immunol 2003; 4: 410-415.

4. Ricci JE, Gottlieb RA, Green DR. Caspase-mediated loss of mitochondrial function and generation of reactive oxygen species during apoptosis. J Cell Biol 2003; 160: 65-75.

5. von Ahsen O, Renken C, Perkins G, Kluck RM, Bossy-Wetzel E, Newmeyer DD. Preservation of mitochondrial structure and function after Bid- or Bax-mediated cytochrome $c$ release. J Cell Biol 2000; 150: 1027-1036.

6. Chipuk JE, Fisher JC, Dillon CP, Kriwacki RW, Kuwana T, Green DR. Mechanism of apoptosis induction by inhibition of the anti-apoptotic BCL-2 proteins. Proc Natl Acad SCi 2008; 105: 20327-20332.

7. Luo X, Budihardjo I, Zou H, Slaughter C, Wang X. Bid, a Bcl2 interacting protein, mediates cytochrome $c$ release from mitochondria in response to activation of cell surface death receptors. Cell 1998; 94: 481-490.

8. Li H, Zhu H, Xu CJ, Yuan J. Cleavage of BID by caspase 8 mediates the mitochondrial damage in the Fas pathway of apoptosis. Cell 1998; 94: 491-501.

9. Kim HR, Chae HJ, Thomas M, Miyazaki T, Monosov A, Monosov E et al. Mammalian dap3 is an essential gene required for mitochondrial homeostasis in vivo and contributing to the extrinsic pathway for apoptosis. FASEB J 2007; 21: 188-196.

10. Meredith JE, Fazeli B, Schwartz MA. The extracellular matrix as a cell survival factor. Mol Biol Cell 1993; 4: 953-961.

11. Frisch SM, Francis $\mathrm{H}$. Disruption of epithelial cell-matrix interactions induces apoptosis. J Cell Biol 1994; 124: 619-626.

12. Frisch SM, Screaton RA. Anoikis mechanisms. Curr Opin Cell Biol 2001; 13: 555-562.

13. Grossmann J. Molecular mechanisms of 'detachment-induced apoptosis-Anoikis'. Apoptosis 2002; 7: 247-260.

14. Miyazaki T, Shen M, Fujikura D, Tosa N, Kim HR, Kon S et al. Functional role of deathassociated protein 3 (DAP3) in anoikis. J Biol Chem 2004; 279: 44667-44672.

15. Lad SP, Yang G, Scott DA, Chao TH, Correia Jda S et al. Identification of MAVS splicing variants that interfere with RIGI/MAVS pathway signaling. Mol Immunol 2008; 45: 2277-2287.

16. Kawai T, Takahashi $\mathrm{K}$, Sato $\mathrm{S}$, Coban $\mathrm{C}$, Kumar $\mathrm{H}$, Kato $\mathrm{H}$ et al. IPS-1, an adaptor triggering RIG-I- and Mda5-mediated type I interferon induction. Nat Immunol 2005; 6 : 981-988.

17. Xu LG, Wang YY, Han KJ, Li LY, Zhai Z, Shu HB. VISA is an adapter protein required for virus-triggered IFN-beta signaling. Mol Cell 2005; 19: 727-740.

18. Meylan E, Curran J, Hofmann K, Moradpour D, Binder M, Bartenschlager R et al. Cardif is an adaptor protein in the RIG-I antiviral pathway and is targeted by hepatitis $\mathrm{C}$ virus. Nature 2005; 437: 1167-1172.

19. Seth RB, Sun L, Ea CK, Chen ZJ. Identification and characterization of MAVS, a mitochondrial antiviral signaling protein that activates NF- $\kappa B$ and IRF 3. Cell 2005; 122 : 669-682.

20. Takahashi K, Kawai T, Kumar H, Sato S, Yonehara S, Akira S. Roles of caspase-8 and caspase-10 in innate immune responses to double-stranded RNA. J Immunol 2006; 176: 4520-4524

21. Kumar H, Kawai T, Kato H, Sato S, Takahashi K, Coban C et al. Essential role of IPS-1 in innate immune responses against RNA viruses. J Exp Med 2006; 203: 1795-1803.

22. Kissil J, Cohen O, Raveh T, Kimchi A. Structure-function analysis of an evolutionary conserved protein, DAP3, which mediates TNF- $\alpha$ and Fas-induced cell death. EMBO J 1999; 18: 353-362.

23. Miyazaki T, Reed JC. A GTP binding adapter protein couples TRAIL receptors to apoptosis-inducing proteins. Nat Immunol 2001; 2: 493-500.

24. Frisch SM. Evidence for a function of death-receptor-related, death-domain-containing proteins in anoikis. Curr Biol 1999; 9: 1047-1049.

25. Rytömaa M, Martins LM, Downward J. Involvement of FADD and caspase-8 signalling in detachment-induced apoptosis. Curr Biol 1999; 9: 1043-1046.

26. Reginato MJ, Mills KR, Paulus JK, Lynch DK, Sgroi DC, Debnath J et al. Integrins and EGFR coordinately regulate the pro-apoptotic protein Bim to prevent anoikis. Nat Cell Biol 2003; 5: 733-740. 